acute coronary syndromes. These findings support the hypothesis that ox-LDL and CRP may play a direct role in promoting the inflammatory component of atherosclerosis. The results suggested that combined use of Oxidised Low Density Lipoprotein and C-reactive protein can predict the acute coronary syndrome. The patient with higher level of ox-LDL and CRP suffer from the higher risk of MACE.

\section{e0452 THE CORONARY ANOMALY: A BRIEF REPORTS OF FIVE CASES ANOMALOUS RIGHT CORONARY ARTERIES}

\section{doi:10.1136/hrt.2010.208967.452}

${ }^{1}$ Xuguang Qin, ${ }^{2}$ Jun Zhu, ${ }^{2}$ Weiguo Xiong, ${ }^{2}$ Chunpeng Lu, ${ }^{2}$ Chengjie Gong. ${ }^{1}$ Department of Cardiology, Hushan Hospital, Fudan University; ${ }^{2}$ Department of Cardiology, First Affiliated Hospital of Tsinghua University

The most common an anomalous right coronary artery (RCA) is that originates from the aortic trunk of ascending artery or left sinus of Valsalva. Herein, we presented five extremely rare cases of anomalous RCA detected incidentally during routine coronary angiography. The first two cases are the anomalous RCA arising separately from the left main stem and proximal of left anterior descending artery (LAD), the third case is the anomalous artery originating from the distal of left circumflex coronary (LCX) artery, and the last two cases are the anomalous RCA deriving from the left sinus of Valsalva.

\section{e0453 EFFECT OF BLOOD PRESSURE LEVELS ON PROGNOSIS IN PATIENTS OF NON ST SEGMENT ELEVATED ACUTE CORONARY SYNDROME WITHOUT PRIOR HISTORY OF HYPERTENSION}

doi:10.1136/hrt.2010.208967.453

Lv Qiang, Liu Xiaohui, Kang Junping, Hu Rong, Wu Jiahui, Ma Changsheng. Beijing Anzhen Hospital

Background Hypertension was an independent risk factor for affecting the prognosis of patients with ACS. But the effect of blood pressure levels at admission on prognosis in ACS patients without history of hypertension was not clear.

Methods The DESIRE-2(Drug-Eluting Stent Impact on Revascularization-2) was a single-center registry of coronary revascularization in our institution between July $1^{\text {st }} 2003$ and Sep $30^{\text {th }} 2005$. The study excluded the patients with prior history of hypertension and myocardial infarction, the patients admitted to hospital with STsegment elevated myocardial infarction and stable coronary artery disease, and the patients without detailed document record. Major adverse cardiac and cerebral events (MACCE) were the combination endpoint of all-cause death, non-fatal myocardial infarction, nonfatal stroke and revascularization.

Results The study enrolled 982 patients and divided into three groups according to the first measurement of blood pressure levels at admission. The 207 patients with blood pressure less than 120/ $80 \mathrm{~mm} \mathrm{Hg}$ defined as normal blood pressure group, 209 patients with blood pressure more than $140 / 90 \mathrm{~mm} \mathrm{Hg}$ as hypertension group, other patients as prehypertension group. Among three groups, there was no difference in age, gender, prior history of diabetes mellitus, coronary revascularization and smoking, body mass index, left ventricular ejection fraction, the levels of glomerular filtration rate, haemoglobin, total cholesterol, low density lipoprotein cholesterin, fasting glucose, glycolated haemoglobin. Severity of coronary artery and utilisation ratio of aspirin, $\beta$ blocker, calcium channel blocker were similar in three groups. More patients taken statins $(67.7 \%, 55.3 \%, 58.3 \%, \mathrm{p}=0.013)$ and ACEI $(41.1 \%, 27.6 \%$,
$27.8 \%, p=0.002)$ during the hospitalisation in hypertension group. Duration of follow-up in the normal, prehypertension and hypertension group was 522 days, 539 days and 523 days respectively. The in-hospital MACCE rates was similar in three groups, but higher follow-up MACCE rates $(11.0 \%, 5.7 \%, 9.5 \%, \mathrm{p}=0.035)$ and followup mortality rates $(2.6 \%, 0.8 \%, 0 \%, \mathrm{p}=0.026)$ in hypertension group. Conclusion The higher blood pressure levels at admission in non ST-segment elevated ACS patients without prior history of hypertension had poorer prognosis, but the history and clinical features were similar to patients with normal blood pressure.

\section{e0454 THE IMPACT OF THERAPY ON 6-MONTHS HEALTH-RELATED QUALITY OF LIFE FOLLOWING ACUTE CORONARY SYNDROME}

doi:10.1136/hrt.2010.208967.454

Yang Lixia, Zhou Yujie. Department Of Cardiology, Beijing Anzhen Hospital, Capital Medical University, Beijing, China

Background Few studies have focused on the change of healthrelated quality of life following acute coronary syndrome, to compare of oral medical therapy, percutaneous coronary intervention and coronary artery bypass grafting. The objective of this study was to identify the changes in Health Related Quality of Life 6 months after discharge from hospital in patients with acute coronary syndrome, and to determine next therapy strategy.

Methods Health Related Quality of Life was assessed in 389 consecutive patients with acute coronary syndrome at the admission and 6 months after discharge which used the Seattle angina questionnaire. To identify the variables associated with the changes, logistic regression models were constructed for five summary dimensions of the Seattle angina questionnaire taking the changes in the score of the dimension as dependent variable.

Results Used the Seattle angina questionnaire scores, all three treatment groups experienced relief of angina at the 6-month visit compared with baseline. At 6 months, in physical limitation, angina stability, angina frequency, percutaneous coronary intervention group and coronary artery bypass grafting group had more significant improvements than Oral medical therapy group $(p<0.001)$. When compared the firstly two group, Coronary artery bypass grafting group had more significant improvements than percutaneous coronary intervention group in angina frequency $(p=0.002)$, treatment satisfaction $(p=0.001)$ and quality life $(p=0.002)$.

Conclusions In patients with acute coronary syndrome, percutaneous coronary intervention group and coronary artery bypass grafting group can provide greater gain in quality life than oral medical therapy, CABG can improve more quality life.

\section{e0455 EFFECT ON LEFT VENTRICULAR FUNCTION AND SAFETY OF HIGH MAINTENANCE DOSE OF CLOPIDOGREL IN PATIENTS WITH ACUTE ANTERIOR MYOCARDIAL INFARCTION UNDERGOING SELECTIVE PCI}

doi:10.1136/hrt.2010.208967.455

Fu Xianghua, Wang Cong, Wang Xuechao, Wang Yanbo, Gu Xinshun, Fan Weize, Wu Weili, Jiang Yunfa. The 2nd Hospital of Hebei Medical University. Shijiazhuang, Hebei China

Objective To assess the beneficial efficacy and safety of the high maintenance dose of clopidogrel in acute anterior myocardial infarction (AMI) patients undergoing selective percutaneous coronary intervention (PCI).

Methods 25 patients were enrolled into this study. These cases were randomly divided into the high maintenance dose group $(n=26$, 